\title{
Boundaries in Digital Planes
}

\author{
EFIM KHALIMSKY \\ College of Staten Island, CUNY, Staten Island, NY 10301
}

RALPH KOPPERMAN

City College of New York, CUNY, New York, NY 10031

PAUl R. MEYer

Lehman College, CUNY, Bronx, NY 10468

\begin{abstract}
The importance of topological connectedness properties in processing digital pictures is well known. A natural way to begin a theory for this is to give a definition of connectedness for subsets of a digital plane which allows one to prove a Jordan curve theorem. The generally accepted approach to this has been a non-topological Jordan curve theorem which requires two different definitions, 4-connectedness, and 8-connectedness, one for the curve and the other for its complement.

In $[\mathrm{KKM}]$ we introduced a purely topological context for a digital plane and proved a Jordan curve theorem. The present paper gives a topological proof of the non-topological Jordan curve theorem mentioned above and extends our previous work by considering some questions associated with image processing:

How do more complicated curves separate the digital plane into connected sets? Conversely given a partition of the digital plane into connected sets,

Ralph Kopperman's Research partly supported by a grant from the PSC-CUNY Award Program.

Bitnet address of Paul R. Meyer: PRMLC@CUNYVM
\end{abstract}

Received: July 1989, Revised: November 1989 
what are the boundaries like and how can we recover them? Our construction gives a unified answer to these questions.

The crucial step in making our approach topological is to utilize a natural connected topology on a finite, totally ordered set; the topologies on the digital spaces are then just the associated product topologies. Furthermore, this permits us to define path, arc, and curve as certain continuous functions on such a parameter interval.

Keywords Jordan curve, linearly ordered topological space, connected ordered topological space, digital plane, computer graphics, finite topological spaces, specialization order, scene, cartoon, screen, separator.

Math. Subject Classification:

54 D 05 Connected and locally connected spaces.

54 F 05 Ordered topological spaces.

68 U 05 Computer graphics; computational geometry.

$68 \mathrm{R} 10$ Graph theory.

68 U 10 Image Processing. 


\section{INTRODUCTION.}

The Jordan curve theorem states that a Jordan curve (simple closed curve) separates the plane into two connected subsets: its inside $I(J)$, and outside $O(J)$. A digital version of this theorem is of central importance in image processing; computer memory space can be conserved by saving only the Jordan curves which outline regions, and a color for each region, rather than the color of each pixel. Since connectedness can be defined in terms of general topology, one way to study this notion is to use a topology on a digital plane. Such a topological approach is used in [KKM], a paper largely based on earlier work by Khalimsky; see [Kh1], [Kh2], and [Kh3].

The present paper extends this topological approach to two-dimensional questions closely related to the Jordan curve theorem and image processing. For example, into how many connected components do more complicated curves carve the plane? In Section 3 we show (Theorem 4) that whenever the plane is partitioned into connected sets, the union of their boundaries has fewer connected components than does its complement.

Secondly, if we are given a partition of the plane into connected sets, when are their boundaries Jordan curves or arcs? Clearly the Jordan curve theorem is useful only to the extent that connected components of the plane can be separated by Jordan curves. In Sections 4 and 5 we show that many pairs of connected components can be so separated, and characterize the curves which separate them.

Our main result in this area relates the following objects (formally introduced in Definition 11): a robust scene is a partition of the plane into sets with connected interiors, each of which is contained in the closure of its interior; a cartoon is a nowhere dense set which is a union of topologically closed separators (a separator is a Jordan curve or an arc which separates the plane into two connected components - see Section 4 for precise definition). Theorem 13 states that for each cartoon $C$ there is a robust scene $\Pi$ 
such that $C$ is the union of the bundaries of the sets in $\Pi$; conversely, given a robust scene $\Pi$ the union of the boundaries of its sets is the unique cartoon $C$ for which the connected components of its complement are the interiors of the sets in $\Pi$.

Graph-theoretic definitions of connectedness which yield a Jordan curve theorem can be found in, among others, [Rf]. Definitions of two kinds of connectedness are required: 4-connectedness and 8-connectedness (for completeness, we restate these definitions in 16). Either can be applied to the curve; the other must then be applied to the background. In Section 5 we discuss the graph-theoretic aproach and give a purely topological proof of the original graph-theoretic Jordan curve theorem (our Theorem 20).

The last Section (5) of this paper also considers how digital planes should be displayed on a computer. In our "open screen" only the topologically open points correspond to pixels (other points are not displayed). This matches the reality in which one does not "see" bounding curves between adjacent regions, but rather deduces them as interfaces of (open) regions. It has the further advantage that the resulting set is topologically homogeneous. Clearly, each robust scene induces a partition of the open screen into 4connected sets. More interesting is the converse (Theorem 19): each such partition of the open screen arises as the trace of a robust scene.

Although this paper looks only at the two-dimensional case, both the topological and graph-theoretic approaches can be extended to three dimensions. For example, [RR], [Re], [KRf], [KR1], [KR2] treat related three-dimensional questions from a graph-theoretic point of view (some methods of topology are used in the last two). In [KMW] a topological three-dimensional Jordan surface theorem is shown.

2 BACKGROUND FROM [KKM].

A COTS (connected ordered topological space) is a connected topological 
space such that among any three points there is one whose deletion leaves the other two in separate components of the remnant. It is shown (Theorem 7) that a COTS topology induces a total order $<$ such that for each nonendpoint $x$, the components of $X-\{x\}$ are $U(x)=\{y: y>x\}$ and $L(x)=$ $\{y: y<x\}$. This order is unique up to inversion. We now describe the topology on a finite COTS (with at least three points) by specifying the minimal open neighborhood $N(p)$ of each point $p$. There are two types of points, and they alternate. If $p$ is of one type, $N(p)=\left\{p^{-}, p, p^{+}\right\}$, where $p^{-}, p^{+}$denote, respectively, the predecessor and successor of $p$ with respect to $<$. By this alternation, $\left\{p^{+}\right\}=N(p) \cap N\left(p^{++}\right)$, is open, so $N\left(p^{+}\right)=\left\{p^{+}\right\}$, further $p \notin N(q)$ if $q \neq p$, thus $X-\{p\}$ is open, so $\{p\}$ is closed. This shows that points alternate between being closed and open. This topology satisfies the $T_{0}$ but not the $T_{1}$ separation property.

If $Y$ is a topological space, a path (respectively arc ) in $Y$ is a continuous (homeomorphic) image of a COTS in $Y ; Y$ is pathwise (arcwise) connected if any two points in $Y$ are the endpoints of a path (an arc) in $Y$. A minimal connected set containing two given points is always an arc which has them as endpoints, thus for finite spaces, connectedness, arcwise connectedness, and pathwise connectedness are equivalent notions. We define the adjacency set $A(x)$ of a point $x \in Y$ by $A(x)=\{y \neq x:\{x, y\}$ is connected $\}$. If $Y$ is finite, $A(x) \cup\{x\}=\mathrm{Cl}(x) \cup N(x)$, where for any $Z \subseteq Y, \mathrm{Cl}(Z)$ denotes the closure of $Z$, and $\mathrm{Cl}(x)=\mathrm{Cl}(\{x\})$. Note that $x \in \mathrm{Cl}(A) \Longleftrightarrow N(x) \cap A \neq \varnothing$, thus $y \in A(x)$ iff $y \in N(x)$ or $x \in N(y)$.

A Jordan curve is a connected space $J$ containing at least 4 elements such that for each $j \in J, J-\{j\}$ is an arc. This is equivalent to the statement that for each $j \in J, A(j) \cap J$ is a two-point discrete set, and $J$ is a nonempty, connected set. A space $X \times Y$ with the product topology, where $X$ and $Y$ are finite COTS with at least 3 elements, is called a digital plane. From now on we restrict our attention to such spaces. A point $(x, y)$ is called 
pure if $\{x\},\{y\}$ are both open or both closed, mixed otherwise. The border $B D(X \times Y)$ of $X \times Y$ is $\{(x, y): x$ or $y$ is an endpoint $\}$. The adjusted border, $A D(X \times Y)$ is $B D(X \times Y)$ with any mixed corner points deleted; corner points are points, both of whose coordinates are endpoints. The adjusted border, unlike the border, is always a Jordan curve.

The following diagrams show the adjacency sets for non-border points in a digital plane. For border (and corner) points the adjacency sets are simply those portions of the sets in the diagrams which are contained in the digital plane.

Adjacency sets for pure and mixed points

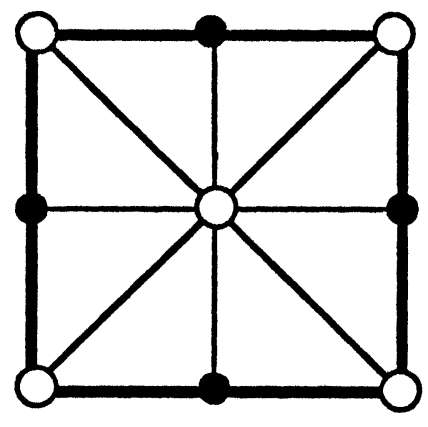

pure point

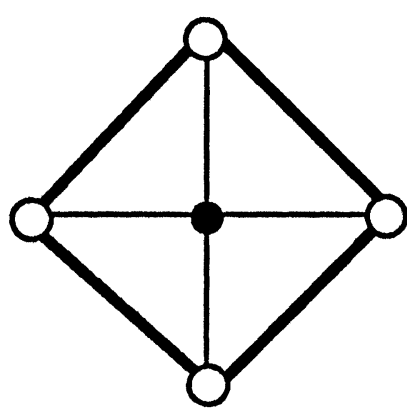

mixed point

$$
\begin{aligned}
& O=\text { pure point } \\
& =\text { mixed point }
\end{aligned}
$$

A sequence $<a_{i}: 1 \leq i \leq n>$ in any topological space is a path iff $\left\{a_{i}, a_{i+1}\right\}$ is connected for each $i$ from 1 to $n-1$. The above diagram enables us to interpret this in a digital plane: $a_{i}$ and $a_{i+1}$ can never be distinct mixed 
points. For an arc in a digital plane, in addition, there can be no "extra connectedness"; thus an arc cannot turn at a mixed point (since the pure points before and after the mixed point would then be connected to each other).

These spaces have a property which will be useful in the sequel: Each point is in the closure of an open point. We will prove this by showing that in a finite $T_{0}$ space each $N(p)$ contains an open point. (Note also that in any topological space, $p$ is in the closure of each point in $N(p)$.)

The proof uses the specialization order (discussed, for example, in $[\mathrm{J}]$ ): (1) For any topological space, $x \leq y$ iff $x \in \mathrm{Cl}(y)$ defines a reflexive, transitive relation. (2) This relation is a partial order (i.e., antisymmetric) iff the space is $T_{0}$ (and then it is called the specialization order). (3) If the space is finite, $x \leq y$ iff $y \in N(x)$ that is, $N(x)=\{y: x \leq y\}$. Thus, $\{y\}$ is open iff $\mathrm{y}$ is maximal with respect to $\leq$. (4) If the space is finite and $T_{0}$, then for each point $p$ we can find a $q \geq p$ which is maximal with respect to $\leq$; for such $q$ by (1)-(3), $N(q)=\{q\}$ and $q \in N(p)$.

As a result of the above, the following are equivalent if $A$ is a subset of a finite $T_{0}$-space:

(i) $A$ is nowhere dense,

(ii) $\operatorname{Int}(A)=\varnothing$

(iii) $A$ contains no open points.

Here (i) $\Rightarrow$ (ii), (ii) $\Rightarrow$ (iii) are clear for arbitrary spaces.

To see that (iii) $\Rightarrow(\mathrm{i}$ ) here, by way of contradiction, if $x \in \operatorname{Int}(\mathrm{Cl}(A))$, then $N(x) \subseteq \mathrm{Cl}(A)$, so an open point $p \in N(x)$ must be in $\mathrm{Cl}(A)$. But then $p \in A$, contradicting (iii).

\section{PARTitions into CONNECTED SETS.}

1 Definition: A scene $\Pi$ in a digital plane is a partition of that plane, all of whose elements are connected. 
For any subset $A$ of the digital plane $X \times Y$, let $\operatorname{Int}(A)$ denote its interior, $A^{c}$ its complement, $\partial A \quad(=\mathrm{Cl}(A)-\operatorname{Int}(A))$ its boundary, and $n(A)$ its (finite) number of elements. If $C$ is an arc, $x, y \in C$, then by $[x, y]$ (or $\left.[x, y]_{C}\right)$ we denote the unique subarc of $C$ with endpoints $x$ and $y$.

2 Lemma: Let $A \cap B=\varnothing$. Then $x \in \partial A \cap \partial B$ iff $N(x)$ meets both $A$ and $B$. Thus if $x \in(\partial A \cap \partial B) \cap(A \cup B)$ then for some $y \in A(x),\{x, y\}$ meets both $A$ and $B$. As a partial converse, if $y \in A(x)$ and $\{x, y\}$ meets both $A$ and $B$, then $\{x, y\}$ meets $\partial A \cap \partial B$.

The first equivalence is clear and the other assertions follow.

3 Lemma: If $J$ is a Jordan curve containing exactly four points, then $J=A(m)$ for some mixed point $m$.

Proof: Every Jordan curve $J$ has an interior $I(J)$; let $m \in I(J)$. Since $J$ has four elements and if $y \in J$ each projection of $J-\{y\}$ is connected, $J$ is contained in a subspace which is the product of 3-point spaces, so $\{m\}=I(J)$. Thus $A(m) \subseteq J$ (otherwise $m$ would be connected to $O(J)$ ). But $A(m)$ contains 4 points for mixed $m, 8$ for pure $m$, and this shows $A(m)=J, m$ mixed.

The material in Sections 4 and 5 is independent of the rest of this Section.

4 Theorem: Let $\Pi$ be a scene in $X \times Y$. Then the number of components of $\cup\{\partial B: B \in \Pi\}$ is (strictly) less than the number of elements of $\Pi$.

The following family of examples shows that the number of components of $C=\cup\{\partial B: B \in \Pi\}$ can be any positive number less than the number of elements of $\Pi$. For $m>j>0$ we give an example of a scene $\Pi$ with $m$ elements such that $C$ has $j$ components, as follows: If $C$ consists of the boundaries of $m-1$ concentric squares not touching the border, then $|\Pi|=m$ and $C$ has $m-1$ components. If $j<m-1$, the example can be modified by 
connecting $m-j$ components of $C$ by a vertical line; notice that this does not change $|\Pi|$.

Before giving the proof of theorem 4, we give a significant special case.

5 Corollary: If $A$ is any connected subset of a digital plane, then $\partial A$ has at most as many components as does its complement $A^{c}$. In particular, if $A$ and $A^{c}$ are connected then so is $\partial A$.

Proof of 5: Notice that $\partial A=\partial A^{c}$, thus it will do to show that $\partial A^{c}=$ $\cup \partial A^{\prime}{ }_{i}$. This results from the more general assertion that if $B$ is the disjoint union $B_{1} \cup \cdots \cup B_{n}$ with $B_{i}$ closed in $B$, then $\partial B=\cup \partial B_{i}$. To see the later, notice that $\partial B=\mathrm{Cl}(B) \cap \mathrm{Cl}\left(B^{c}\right)=\left[\cup \mathrm{Cl}\left(B_{i}\right)\right] \cap \mathrm{Cl}\left(B^{c}\right)=\cup\left[\mathrm{Cl}\left(B_{i}\right) \cap\right.$ $\left.\mathrm{Cl}\left(B^{c}\right)\right] \subseteq \cup\left[\mathrm{Cl}\left(B_{i}\right) \cap \mathrm{Cl}\left(B_{i}^{c}\right)\right]=\cup \partial B_{i}$.

But if $x \in \mathrm{Cl}\left(B_{i}^{c}\right)$ then $x \in \mathrm{Cl}(B)$ or $x \in \mathrm{Cl}\left(B_{j}\right)$ for some $j \neq i$. Also if $x \in \mathrm{Cl}\left(B_{i}\right) \cap \mathrm{Cl}\left(B_{j}\right)$ then $x \in B^{c} \subseteq \mathrm{Cl}\left(B^{c}\right)$, otherwise $\mathrm{Cl}\left(B_{i}\right) \cap \mathrm{Cl}\left(B_{j}\right) \cap B \neq$ $\varnothing$, contradicting the fact that each $B_{j}$ is closed in $B$. Since $\operatorname{Cl}\left(B_{i}\right) \subseteq \mathrm{Cl}(B)$, $x \in \partial B$, completing the proof.

Proof of 4: We proceed by induction, first noting that the result is clear if $n(\Pi)=1$. Otherwise suppose for the moment that there are $A, B \in \Pi$ such that $(\partial A \cap \partial B) \cap(A \cup B)$ consists of one non-empty component. Notice that in this situation, $A \cup B$ is connected, for if $\varnothing \neq F \subseteq(\partial A \cap \partial B) \cap(A \cup B)$, then $A \subseteq A \cup F \subseteq \mathrm{Cl}(A), B \subseteq B \cup F \subseteq \mathrm{Cl}(B)$, so the sandwiched sets are connected, and since they meet, their union, $A \cup B$ is connected. Also, $\partial(A \cup B) \cup(\partial A \cap \partial B)=\partial A \cup \partial B$, for:

$x \in \partial(A \cup B)$ iff $N(x)$ meets $A \cup B$ and another member of $\Pi$, $x \in \partial A \cap \partial B$ iff $N(x)$ meets $A$ and $B$,

the disjunction of these two is equivalent to the condition:

$N(x)$ meets $A$ and another element of $\Pi$ or $B$ and another element of $\Pi$, 
i.e., that $x \in \partial A \cup \partial B$. Also note that if $x \in(\partial A \cup \partial B)-(A \cup B)$, then for some $D \in \Pi-\{A, B\}, x \in D$ and $N(x)$ meets $D^{c}$, so $x \in \cup\{\partial D: D \in$ $\Pi-\{A, B\}\}$. Thus, letting $\Pi^{*}=(\Pi \cup\{A \cup B\})-\{A, B\}, \cup\{\partial D: D \in \Pi\}=$ $\partial A \cup \partial B \cup \bigcup\{\partial D: D \in \Pi-\{A, B\}\}=(\partial A \cap \partial B) \cup\left(\bigcup\left\{\partial D: D \in \Pi^{*}\right\}=\right.$ $[(\partial A \cap \partial B) \cap(A \cup B)] \cup\left(\bigcup\left\{\partial D: D \in \Pi^{*}\right\}\right)$, so its number of components is less than $1+n\left(\Pi^{*}\right)=n(\Pi)$.

Thus to complete the proof, it will do to show 7 (b) below; 7 (a) is used in its proof.

6 Definition: $A$ joining pair for $A, B$ is a pair of $\operatorname{arcs} C \subseteq A$ with endpoints $x, y, C^{\prime} \subseteq B$ with endpoints $y^{\prime}, x^{\prime}$ such that $\left\{x, x^{\prime}\right\}$ and $\left\{y, y^{\prime}\right\}$ are connected sets meeting distinct components of $\partial A \cap \partial B$. The endpoints of $C, C^{\prime}$ are called the endpoints of the joining pair. A joining Jordan curve for $A, B$ is a Jordan curve which is the union of a joining pair for $A, B$.

\section{Lemma:}

(a) If $A, B$ are disjoint sets, and $C, C^{\prime}$ is a joining pair for $A, B$ whose endpoints meet distinct components of $\partial A \cap \partial B$, then there is a joining Jordan curve $J$ for $A, B$ such that $J \subseteq C \cup C^{\prime}$.

(b) If $\Pi$ is a scene in $X \times Y$ containing more than one element, then for some $A, B \in \Pi,(\partial A \cap \partial B) \cap(A \cup B)$ consists of one non-empty component.

Proof: (a) From among connected $\left\{v, v^{\prime}\right\},\left\{w, w^{\prime}\right\}$ which meet $(\partial A \cap \partial B) \cap$ $(A \cup B)$ at distinct components, choose such that $C^{*}=[v, w]$ is as short as possible, and given that, such that $C^{* \prime}=\left[w^{\prime}, v^{\prime}\right]$ is as short as possible. To show that their union $J$ is a Jordan curve, it will do to show that if $z \in J$, $A(z) \cap J$ contains exactly two elements. First, note that if $z \in C^{*}-\{v, w\}$, then $A(z) \cap C^{*} \subseteq A(z) \cap J$; further, $\left\{v^{\prime}\right\} \cup\left(A(v) \cap C^{*}\right) \subseteq A(v) \cap J$, so $A(v) \cap J$ contains at least two elements, and similarly so does $A(w) \cap J$. This shows that if $z \in C^{*}$ then $A(z) \cap J$ has at least two elements, and a 
similar argument shows the same if $z \in C^{* \prime}$. Thus if $J$ were not a Jordan curve, there would be an element $z$ of $C^{*}$, or no element of $C^{*}$ but a $z \in C^{* \prime}$, such that $A(z) \cap J$ contained at least 3 elements. In the first case, since $C^{*}$ is an arc, $A(z) \cap C^{*}$ has at most two elements, so $A(z) \cap C^{* \prime} \neq \varnothing$, and a similar argument shows the second case to be impossible. Thus let $z \in C^{*}$, $z^{\prime} \in C^{* \prime} \cap A(z)$ (and note that if $z \in\{v, w\}$ then $z^{\prime} \notin\left\{v^{\prime}, w^{\prime}\right\}$ ). Since $\left\{z, z^{\prime}\right\}$ meets both $A$ and $B$ and $z^{\prime} \in A(z),\left\{z, z^{\prime}\right\}$ must meet $(\partial A \cap \partial B) \cap(A \cup B)$, and the component at which it meets $(\partial A \cap \partial B) \cap(A \cup B)$ must be distinct from either the component meeting $\left\{v, v^{\prime}\right\}$ or that meeting $\left\{w, w^{\prime}\right\}$. If it is distinct from that meeting $\left\{v, v^{\prime}\right\}$, then the segments $[v, z]$ of $C^{*}$ from $v$ to $z$ and $\left[z^{\prime}, v^{\prime}\right]$ of $C^{* \prime}$ are arcs in $A, B$ respectively, joining connected pairs which intersect distinct components of $(\partial A \cap \partial B) \cap(A \cup B)$, and $[v, z]$ is shorter than $C^{*}$ or $\left[z^{\prime}, v^{\prime}\right]$ shorter than $C^{* \prime}$, contradicting minimality. (A similar argument holds if it is distinct from that meeting $\left\{w, w^{\prime}\right\}$.) Since $C^{*}, C^{* \prime}$ are clearly a joining pair for $A, B$, this completes the proof of (a).

If (b) fails then for each $A, B \in \Pi$, there are distinct components of ( $\partial A \cap$ $\partial B) \cap(A \cup B)$. By 2 and (a) we may find a joining Jordan curve for $A, B, J=$ $C \cup C^{\prime}$ with endpoints $x, y \in A, x^{\prime}, y^{\prime} \in B$; we also require that $I(J)$ contain as few elements as possible for a joining Jordan curve for any $A^{\prime}, B^{\prime} \in \Pi$. In the following paragraph, we show the existence of $z \in C-\{x, y\}$ (or in $\left.C^{\prime}-\left\{y^{\prime}, x^{\prime}\right\}\right)$ for which $A(z) \cap I(J)$ meets some $\left.D \in \Pi-\{A, B\}\right)$.

First note that if $z \in C-\{x, y\}$, then $A(z) \cap I(J)$ does not meet $B$ : Otherwise find $z^{\prime} \in A(z) \cap I(J),\left\{z, z^{\prime}\right\}$ intersecting $A$ and $B$, thus $(\partial A \cap$ $\partial B) \cap(A \cup B)$ at a component distinct from that meeting $\left\{x, x^{\prime}\right\}$ or that meeting $\left\{y, y^{\prime}\right\}$. In the first case let $D$ be an $\operatorname{arc}$ in $B$ connecting $z^{\prime}$ to $x$; $D$ meets $C^{\prime}$ at a first place, $w^{\prime}$, and let $D^{\prime}$ be an arc in the connected set $\left[z^{\prime}, w^{\prime}\right] \cup\left[w^{\prime}, x\right]$ joining $z^{\prime}$ to $x$. But then $[x, z], D^{\prime}$ are a joining pair for $A, B$, so, by (a), find a joining Jordan curve for $A, B, J^{\prime}=C^{*} \cup C^{* \prime} \subseteq[x, z] \cup D^{\prime}$; note that $J^{\prime} \subseteq J \cup I(J)$, thus $I\left(J^{\prime}\right)$ is a connected set which meets $I(J)$ 
but not $J$ thus $I\left(J^{\prime}\right) \subseteq I(J)$, and since $z^{\prime} \in I(J)-I\left(J^{\prime}\right), I\left(J^{\prime}\right)$ is properly smaller, contradicting minimality. A similar argument holds in the second case. Next note that for $z \in C-\{x, y\}, z, I(J) \cap A(z) \nsubseteq A$, since otherwise $(C-\{z\}) \cup(I(J) \cap A(z))$ is a connected subset of $A$ containing $x$ and $y$ so there is an $\operatorname{arc} C^{*}$ from $x$ to $y$ in $(C-\{z\}) \cup(I(J) \cap A(z))$, and since $x, y$ are in distinct components of $C-\{z\}, C^{*}$ must meet $I(J) \cap A(z)$, say at $s$. We apply (a) to obtain $J^{*} \subseteq C^{*} \cup C^{\prime} \subseteq J \cup I(J)$ a joining Jordan curve for $A, B . I\left(J^{*}\right) \subseteq I(J)-\{s\}$, contradicting the minimality of $I(J)$. Finally, $J \neq\left\{x, y, y^{\prime}, x^{\prime}\right\}$, for otherwise by $3, J=A(m)$ for some mixed point $m$. But then its two closed points, say $\left\{x, y^{\prime}\right\}$ meet distinct components of $(\partial A \cap \partial B) \cap(A \cup B)$. But $m \in \mathrm{Cl}(y) \cap \mathrm{Cl}\left(x^{\prime}\right) \subseteq \mathrm{Cl}(A) \cap \mathrm{Cl}(B)$, so $m \in D$ for some $D \in \Pi-\{A, B\}$, in which case we're done, or $\left\{x, m, y^{\prime}\right\}$ is a connected subset of $(\partial A \cap \partial B) \cap(A \cup B)$, contradicting the fact that $x, y^{\prime}$ are in distinct components of $(\partial A \cap \partial B) \cap(A \cup B)$.

Let $J, z, C, A, D$ be as in the previous paragraph, $z^{\prime} \in D \cap A(z) \cap I(J)$. We show that $(\partial A \cap \partial D) \cap(A \cup D)$ consists of one non-empty component, completing the proof of (b): It is non-empty since $z \in A$ and $A(z)$ meets $D$. But if it had two components, we could find $\operatorname{arcs} E \subseteq A$, from $v$ to $w, E^{\prime} \subseteq D$, from $w^{\prime}$ to $v^{\prime}$, with $\left\{v, v^{\prime}\right\},\left\{w, w^{\prime}\right\}$ connected. Since $D$ meets $I(J)$ but not $J$ and $D$ is connected, $D \subseteq I(J)$, thus $E^{\prime} \subseteq I(J)$. Further note that we may require that $E \subseteq J \cap I(J)$, for otherwise replace $E$ by an arc connecting $v$ to $w$ in the connected $[v, s]_{E} \cup[s, t]_{C} \cup[t, w]_{E}$, where $s$ and $t$ are respectively the first and last points in $E$ which are in $C$ (if $E$ doesn't meet $C$ then $E$ doesn't meet $J$ but does meet $\mathrm{Cl}(I(J))$ and is connected, thus is contained in $I(J))$. Using (a) obtain a joining Jordan curve $K \subseteq\left(E \cup E^{\prime}\right) \cap(J \cup I(J))$, thus $I(K) \subseteq I(J)$. But $\varnothing \neq K \cap D \subseteq I(J)-I(K)$, contradicting the minimality of $I(J)$. (A similar proof shows $(\partial D \cap \partial B) \cap(D \cup B)$ consists of one non-empty component if $z \in C^{\prime}$.)

8 Example ("Comb space"): We exhibit a connected open set $A$ with 
connected complement, whose boundary isn't an arc or Jordan curve. Let $X=Y=\{1,2,3,4\}$ with minimal neighborhoods $\{1\},\{1,2,3\},\{3\},\{3,4\}$. In $X \times Y$ let $A=(\{1\} \times Y) \cup(\{1,2,3\} \times\{1,3\}) ; A$ is a union of products of open sets, thus is open. By splitting $\{1,3\}$ we see $A$ a union of three connected sets, one of which meets the other two, thus connected, and a similar look shows $A^{c}$ to be connected. Finally, $A$ is dense since $\operatorname{Cl}(A) \supseteq$ $\mathrm{Cl}((3,3))=\{2,3,4\} \times\{2,3,4\}$ so $\{(4,1)\}=A^{c}-\mathrm{Cl}((3,3))$, and $(4,1) \in$ $\mathrm{Cl}((3,1)) \subseteq \mathrm{Cl}(A)$ as well. Thus $\partial A=\mathrm{Cl}(A)-\operatorname{Int}(A)=X \times Y-A=A^{c}$, and $(4,2) \in A^{c}, A((4,2)) \cap A^{c}=\{(4,1),(4,3),(3,2)\}$ contains over two points.

9 Computer application: Suppose a scene, $\Pi$, is entered into computer memory (ie., each point of $X \times Y$ is assigned the index of the member of $\Pi$ containing it). To find the boundaries of members of $\Pi$, first recall that $z$ is a boundary point iff $N(z)$ meets distinct elements of $\Pi$ (thus there are no open boundary points). Let $x_{i} \in A_{i}$, and let $B_{i j}$ be a connected set containing $x_{i}$ and $x_{j}$ (for example, $B_{i j}$ could be the union of a horizontal line segment containg $x_{i}$ with a vertical line segment containing $x_{j}$ which meets it). For each $i, j$ :

(i) Follow $B_{i j}$ until $B_{i j}$ crosses from one partition element to another at a boundary point not yet found. Beginning with that point $z$ :

(ii) Check all the non-open points of $A(z)$ (there are 2 of these for mixed $z, 4$ for closed $z$ ) which have not yet been checked, to see whether they are boundary points. Let one of these boundary points be the next $z$ and store the rest for furture use. If no new boundary points are found at a particular $z$, go back to the most recently stored unused boundary point and use it as the next $z$. When there are no more unused points, you have found a component of the union of the boundaries. Then go back to (i), continuing to follow the same $B_{i j}$ which was being followed until it is exhausted; then go on to the next. 
It may help to keep count of the number of boundary components found, since there are at most $n$ by theorem 4 . But the examples given after the statement of theorem 4 show that there can be any positive number smaller than $n$. (The scene with one element has empty boundary.)

\section{Cartoons AND Robust SCENES.}

In the previous Section, particularly Example 8, we left open the question of what sorts of pictures can be "drawn" by making outlines and coloring in the regions so described; note that only connected such regions need be considered. We find it convenient to let $J$ be either a Jordan curve not meeting $B D(X \times Y)$ or an arc with at least three points which meets $B D(X \times$ $Y)$ at precisely its endpoints. In [KKM] we showed that if $J$ is of either of these types, then $X \times Y-J$ falls into two connected components. We call such a $J$ a separator, and generalize the notation $I(J), O(J)$ for the components as follows: (using natural orders on $X, Y)$ order $B D(X \times Y)$, and call the component containing the first point in $B D(X \times Y)-J O(J)$, the other $I(J)$. A bit of consideration shows that not every separator $J$ is the boundary of $I(J)$, but our next result shows just when this does happen.

10 Proposition: The following are equivalent for $J$ a separator:

(a) $J=\partial(I(J))$

(b) $J$ contains no open points,

(c) $J$ is closed,

(d) $J$ is nowhere dense,

(e) $I(J)$ and $O(J)$ are open,

(f) $I(J) \cup O(J)$ is dense.

Proof: $(\mathrm{a}) \Rightarrow(\mathrm{c})$ holds since boundaries are closed.

(c) $\Rightarrow$ (b): If $p \in J$, then (by [KKM 17(a)]) $A(p) \cap J$ contains at most two points (exactly two for non-border $p$ ). But if $p$ is open, $J$ closed, $A(p) \cup\{p\}=$ $\mathrm{Cl}(p) \subseteq \mathrm{Cl}(J)=J$, and $A(p)$ contains more than two elements. 
For (b) $\Rightarrow$ (a) we first show that if $x \in J$ is closed or mixed, then $N(x)$ meets $I(J)$, thus $J \subseteq \mathrm{Cl}(I(J))$; since $J$ doesn't meet $I(J)$ it doesn't meet $\operatorname{Int}(I(J))$ so $J \subseteq \partial(I(J))$.

But $A(x)=A D\left(X^{\prime} \times Y^{\prime}\right)$ where $X^{\prime}, Y^{\prime}$ are the projections of $A(x)$ and usually contain three points (though if $x$ is an endpoint, that projection will contain two points). In [KKM] it was shown that $A(x)-J=A D\left(X^{\prime} \times Y^{\prime}\right)-J$ meets each component of $X \times Y-J$. But if $x$ is closed, $N(x) \supseteq A(x)$ and thus meets $I(J)$, and if $x$ is mixed then $N(x) \supseteq A(X)-\mathrm{Cl}(x) \supseteq A(x)-J$, so again $N(x)$ meets $I(J)$.

Next note that since $X \times Y-J$ contains only two components, $I(J)$ and $O(J)$, each of these is relatively open in $X \times Y-J$. If $J$ is closed then $X \times Y-J$ is open in $X \times Y$, thus $I(J), O(J)$ are open in $X \times Y$, so $I(J) \cup J=X \times Y-O(J)$, a closed set and $I(J)=\operatorname{Int}(I(J))$. Thus $\partial(I(J))=\mathrm{Cl}(I(J))-\operatorname{Int}(I(J)) \subseteq(J \cup I(J))-I(J)=J$, completing the proof.

(c) $\Rightarrow(\mathrm{e})$ was shown in the previous paragraph; (e) $\Rightarrow$ (c) since $J$ is the complement of $I(J) \cup O(J)$.

Since $J$ is the complement of $I(J) \cup O(J),(\mathrm{b}) \Longleftrightarrow$ (d) $\Longleftrightarrow$ (f) holds by the last paragraph of Section 2.

11 Definition: $\mathrm{A}$ set $A$ is regular if $\mathrm{Cl}(A)=\mathrm{Cl}(\operatorname{Int}(A))$ and $\operatorname{Int}(A)=$ $\operatorname{Int}(\mathrm{Cl}(A))$. A robust scene is a scene $\Pi$, each of whose elements has connected interior and is contained in the closure of its interior. A cartoon is a finite union of closed separators.

Note that as a finite union of closed, nowhere dense sets, each cartoon is closed and nowhere dense. Here are some easy equivalences, given without proof:

$$
\begin{aligned}
& A \text { is regular } \Longleftrightarrow \\
& A^{c} \text { is regular } \Longleftrightarrow \\
& \partial A=\partial \operatorname{Int}(A)=\partial \operatorname{Cl}(A) \Longleftrightarrow
\end{aligned}
$$




\section{$\operatorname{Int}(\mathrm{Cl}(A)) \subseteq A$ and $A \subseteq \mathrm{Cl}(\operatorname{Int}(A))$.}

In general, $B$ is regular if and only if for some $A, \operatorname{Int}(\operatorname{Cl}(A)) \subseteq B \subseteq$ $\mathrm{Cl}(\operatorname{Int}(A))$.

Note that each partition of the plane into sets with connected interior, each of which is contained in the closure of that interior, is a robust scene, since any subset of the closure of a connected set is connected. Also note that robust scenes are collections of regular sets. In fact, if $\Pi$ is any (finite) partition of $X \times Y$, and $A \subseteq \mathrm{Cl}(\operatorname{Int}(A))$ for each $A \in \Pi$, then $\Pi$ is a set of regular sets, for $\operatorname{Int}(\mathrm{Cl}(A))=\left(\operatorname{Cl}\left(\operatorname{Int}\left(A^{c}\right)\right)\right)^{c}=X \times Y-\cup\{\mathrm{Cl}(\operatorname{Int}(B)): B \in$ $\Pi-\{A\}\} \subseteq X \times Y-\cup(\Pi-\{A\})=A$.

12 Theorem: The following are equivalent:

(i) $A \neq \varnothing, X \times Y, A$ is regular and $\operatorname{Int}(A), \operatorname{Int}\left(A^{c}\right)$ are connected,

(ii) $\partial A$ is a closed separator $J$ for which $I(J) \subseteq A \subseteq I(J) \cup J$ or $O(J) \subseteq$ $A \subseteq O(J) \cup J$.

Proof: (i) $\Rightarrow$ (ii) We have shown that $\partial A \neq \varnothing$ is connected, thus it will suffice to show that for each non-border point $x$ of $\partial A, n(A(x) \cap A)=2$. If $x \in \partial A$ then $\mathrm{Cl}(x) \subseteq \mathrm{Cl}(A), \mathrm{Cl}(x) \subseteq X \times Y-\operatorname{Int}(A)$ so $\mathrm{Cl}(x) \subseteq \partial A$. If $x$ is mixed then $\mathrm{Cl}(x)$ consists of $x$ and the two closed points in $A(x)$, so these are in $\partial A$, and by previous comments, the other two points of $A(x)$, being open, cannot belong to $\partial A$. Thus $A(x) \cap \partial A$ is just the two closed points. For any $x \in \partial A, x \in \mathrm{Cl}(A)=\mathrm{Cl}(\operatorname{Int}(A))$ so for some open $y \in A, x \in \mathrm{Cl}(y)$.

For closed $x$ we now show $A(x) \cap \operatorname{Int}(A)$ to be connected. If not, let $C$ be an arc of minimal length connecting distinct components of this set through the connected $\operatorname{Int}(A)$. By this minimality, $C$ meets $\operatorname{Int}(A) \cap A(x)$ precisely at its endpoints, say $v, w$. Thus $K=C \cup\{x\}$ is a Jordan curve $(A(x) \cap K=\{v, w\}, A(v) \cap K=\{x\} \cup(A(v) \cap C), A(w) \cap K=\{x\} \cup(A(w) \cap C)$, and for $t \in C-\{v, w\}, t \notin A(x)$ so $A(t) \cap K=A(t) \cap C$, a set with two elements). But then $\mathrm{Cl}\left(A^{c}\right) \cap A(x)$ meets $I(K)$ and $O(K)$, since otherwise there would be an arc in $A(x) \cap \operatorname{Int}(A)$ connecting $v$ to $w$. But this contradicts 
that $\operatorname{Int}\left(A^{c}\right)$ is connected, thus so is $\mathrm{Cl}\left(A^{c}\right)=\mathrm{Cl}\left(\operatorname{Int}\left(A^{c}\right)\right)$ : no arc could join $I(K)$ to $O(K)$ without meeting $K \subseteq \operatorname{Int}(A) \cup\{x\} \subseteq \mathrm{Cl}(A)$.

We now use the connectedness of $A(x) \cap \operatorname{Int}(A)$ to show that exactly two points of $A(x)$ are in $\partial A$ : Since $A(x)$ is a Jordan curve, its only connected subsets (other than $A(x)=N(x), \varnothing$, which are ruled out since $x \in \partial A$ ) are arcs; thus $A(x) \cap \operatorname{Int}(A), A(x) \cap \operatorname{Int}\left(A^{c}\right)$ are such, and are open, disjoint subsets of the connected set $A(x)$ whose union contains all four open points of $A(x)$. Assume $n\left(A(x) \cap \operatorname{Int}\left(A^{c}\right)\right) \leq n\left(A(x) \cap \operatorname{Int}\left(A^{c}\right)\right.$ ) (interchanging $A$ and $A^{c}$ if necessary). Since $A$ is regular, $\partial A=\mathrm{Cl}(\operatorname{Int}(A))-\operatorname{Int}(\mathrm{Cl}(A))=$ $\mathrm{Cl}(\operatorname{Int}(A)) \cap \mathrm{Cl}\left(\operatorname{Int}\left(A^{c}\right)\right)$, so a mixed point $w \in A(x)$ is in $\partial A$ iff $N(w)$ meets both $\operatorname{Int}(A)$ and $\operatorname{Int}\left(A^{c}\right)$; ie., iff the corners of the side of the square $A(x)$ containing $w$ are in opposites of $A, A^{c}$. If $n(A(x) \cap \operatorname{Int}(A))=1$ then for this reason, $A(x) \cap \partial A$ contains exactly the two mixed points adjacent to that corner. If $n(A(x) \cap \operatorname{Int}(A))=2$ then since this set is an arc, the two open corner points so described can't be opposite, so are ends of one side of the square; clearly the mixed point between them is not in $\partial A$ nor is the mixed point on the opposite side, but the two other mixed points have one corner from $A$ and one from $A^{c}$ on their sides, and are in $\partial A$.

Thus let $J=\partial A$; since $\varnothing \neq A \subseteq \mathrm{Cl}(\operatorname{Int}(A))$, $\operatorname{Int}(A) \neq \varnothing$, so since $\mathrm{Cl}(I(J)) \cup \mathrm{Cl}(O(J))=X \times Y$, Int $(A)$ must meet $I(J)$ or $O(J)$; assume the first. Then since $\operatorname{Int}(A)$ is connected and $\operatorname{Int}(A) \cap J=\varnothing, \operatorname{Int}(A) \subseteq I(J)$; since $I(J)$ is connected and $I(J) \cap J=\varnothing, \operatorname{Int}(A) \supseteq I(J)$. Since $A$ is regular, $I(J) \subseteq A \subseteq \mathrm{Cl}(A)=\mathrm{Cl}(\operatorname{Int}(A))=\mathrm{Cl}(I(J))=I(J) \cup J$.

For the converse, assume with no loss of generality that $I(J) \subseteq A \subseteq$ $I(J) \cup J$. Notice that by $10, J=\partial I(J)$, (and since $J$ is non-empty and $X \times Y$ is connected, $\varnothing \neq I(J) \subseteq A)$ so $\mathrm{Cl}(I(J))=I(J) \cup J$ and $I(J)$ is open, so $I(J)=\operatorname{Int}(I(J)) \subseteq \operatorname{Int}(A) \subseteq \operatorname{Int}(\operatorname{Cl}(A))$. Also $\mathrm{Cl}(\operatorname{Int}(A)) \supseteq \operatorname{Cl}(I(J))=$ $I(J) \cup J \supseteq A$ and $I(J)=(J \cup O(J))^{c}=(\mathrm{Cl}(O(J)))^{c}=\operatorname{Int}(I(J) \cup J) \supseteq$ $\operatorname{Int}(\mathrm{Cl}(A))$. Thus $\operatorname{Int}(\mathrm{Cl}(A))=I(J) \subseteq A$ and $A$ is regular, so further $\partial A=$ 
$\partial \operatorname{Int}(A)=\partial I(J)=J($ by $10(\mathrm{a}))$. It was shown in $[\mathrm{KKM}]$ that $I(J)=\operatorname{Int}(A)$ and $O(J)=\operatorname{Int}\left(A^{c}\right)$ are connected.

13 Theorem Let $C$ be a cartoon. Then there is a robust scene $\Pi$ such that $C=\cup\{\partial A: A \in \Pi\}$. Further, if $\Pi^{\prime}$ is any other such robust scene, then $\{\operatorname{Int}(A): A \in \Pi\}=\left\{\operatorname{Int}(A): A \in \Pi^{\prime}\right\}$.

Conversely, if $\Pi$ is a robust scene then $C=\cup\{\partial A: \in \Pi\}$ is a cartoon.

In Example 8 we showed a scene which does not give rise to a cartoon. We need the following lemma to prove Theorem 13:

14 Lemma: Let $\Pi$ be a robust scene.

(a) if $A, B \in \Pi$ then $A \cup B$ is regular.

(b) For $A, B \in \Pi$ the following are equivalent:

(i) $\operatorname{Int}(A \cup B)$ is connected.

(ii) $(\Pi-\{A, B\}) \cup(\{A \cup B\})$ is a robust scene.

(iii) There is a mixed point in $\partial A \cap \partial B$.

(c) If $A$ is regular and $c \in \partial A-B D(X \times Y)$ is closed, then $A(c) \cap \partial A$ contains at least two points, consists completly of mixed points, and is not contained in $B D(X \times Y)$.

(d) If $m \in \partial A$ is mixed and $c \in A(m)$ is closed then $c \in \partial A$ is contained in every closed arc or Jordan curve $C$ such that $m \in C \subseteq \partial A$.

Proof: (a) Since $A, B$ are regular, $A \cup B \subseteq \mathrm{Cl}(\operatorname{Int}(A)) \cup \mathrm{Cl}(\operatorname{Int}(B))=$ $\mathrm{Cl}(\operatorname{Int}(A) \cup \operatorname{Int}(B)) \subseteq \mathrm{Cl}(\operatorname{Int}(A \cup B))$. Also if $x \in \operatorname{Int}(\mathrm{Cl}(A \cup B))$ then $N(x) \subseteq \mathrm{Cl}(A \cup B)=\mathrm{Cl}(A) \cup \mathrm{Cl}(B)$, so each open point of $N(x)$ is in $\mathrm{Cl}(A)$ or $\mathrm{Cl}(B)$, thus in $A$ or $B$ (if an open $y \in \mathrm{Cl}(C)$ then the open $\{y\}$ meets $C$ so $y \in C)$. By the regularity of the other $C \in \Pi$, since $\operatorname{Int}(C) \cap N(x)=\varnothing$, $x \notin \mathrm{Cl}(\operatorname{Int}(C)) \supseteq C$ so $x \in A \cup B$.

(b) (i) $\Longleftrightarrow$ (ii) since by (a) $(\Pi-\{A, B\}) \cup\{A \cup B\}$ is a partition of $X \times Y$ into regular sets. 
(iii) $\Rightarrow$ (i): Let $m \in \partial A \cap \partial B$ be mixed. Then by the regularity of $A, B, m \in \mathrm{Cl}(\operatorname{Int}(A)), \mathrm{Cl}(\operatorname{Int}(B))$, so one each of the open points in $N(m)$ must be in $A, B$. By regularity of the remaining elements of $\Pi, m \in A \cup B$ (as in the proof of (a)). Thus $N(m) \subseteq A \cup B$ so $N(m) \subseteq \operatorname{Int}(A \cup B$ ). If $x \in \operatorname{Int}(A \cup B)$ then $N(x) \subseteq A \cup B$ so let $p \in N(x)$ be open; then $p \in A$ or $p \in B$ so $p \in \operatorname{Int}(A)$ or $p \in \operatorname{Int}(B)$. In the first case, since $\operatorname{Int}(A)$ is connected and meets the connected $N(m), N(x)$ we have $m, x \in N(x) \cup \operatorname{Int}(A) \cup N(m) \subseteq$ $\operatorname{Int}(A \cup B)$, in the second we similarly have $m, x \in N(x) \cup \operatorname{Int}(B) \cup N(m) \subseteq$ $\operatorname{Int}(A \cup B)$. Thus in either case, $x$ is in the same component of $\operatorname{Int}(A \cup B)$ as $m$, so $\operatorname{Int}(A \cup B)$ is the component of $m$ in it, thus connected.

(i) $\Rightarrow($ iii): Let $x \in \operatorname{Int}(A), y \in \operatorname{Int}(B)$. Then by (i) there is an $\operatorname{arc} C \subseteq$ Int $(A \cup B)$ with endpoints $x \in A, y \in B$, so (by 2 applied to the last point of $C \cap A$ and its successor in $C), C \cap(\partial A \cap \partial B) \neq \varnothing$. Thus let $z \in C \cap(\partial A \cap \partial B)$. Then $z \in C \subseteq \operatorname{Int}(A \cup B)$ so $N(z) \subseteq A \cup B$ and $N(z) \cap \operatorname{Int}(A) \neq \varnothing \neq N(z) \cap \operatorname{Int}(B)$. If $z$ is mixed, we're done and by $10, z$ can't be open, so $z$ is closed, and $N(z)$ must be a $3 \times 3$ "square", and one of its sides must meet both $\operatorname{Int}(A)$ and $\operatorname{Int}(B)$, so the mixed point on that side must be in $\partial A \cap \partial B$.

(c) Since $A$ is regular, so is $A^{c}$, so $\partial A=\mathrm{Cl}(A) \cap \mathrm{Cl}\left(A^{c}\right)=\mathrm{Cl}(\operatorname{Int}(A)) \cap$ $\mathrm{Cl}\left(\operatorname{Int}\left(A^{c}\right)\right)$. Among the four open "corner points" of the "square" $N(c)$ are some in $A$ and some in $A^{c}$, so at least two "sides" of $N(c)$ meet both $A$ and $A^{c}$. The mixed points on those sides are in $\mathrm{Cl}(A) \cap \mathrm{Cl}\left(A^{c}\right)=\partial A$.

(d) If $m \in \partial A=\mathrm{Cl}(A) \cap \mathrm{Cl}\left(A^{c}\right)$, then $\mathrm{Cl}(m) \subseteq \mathrm{Cl}(A) \cap \mathrm{Cl}\left(A^{c}\right)$, so $\mathrm{Cl}(m)-\{m\} \subseteq A(m) \cap \partial A$. Since the remaining points of $A(m)$ are open, they can't be in $\partial A$, so $\mathrm{Cl}(m)-\{m\}=A(m) \cap \partial A$.

Proof of 13: Given $C$, let $P$ be an enumeration of the set of connected components of $X \times Y-C, P=\left\{Q_{1}, \ldots, Q_{n}\right\}$. Then $P$ is a set of connected sets which are open (as in the discussion of $I(J), O(J)$ in the proof of 10 , (b) $\Rightarrow(\mathrm{a}))$. Also, $\mathrm{Cl}(\cup P)=X \times Y-\operatorname{Int}(C)=X \times Y$, so $\cup P$ is dense. For 
$1 \leq j \leq n$ let $A_{j}=\mathrm{Cl}\left(Q_{j}\right)-\cup_{k<j} \mathrm{Cl}\left(Q_{k}\right), \Pi=\left\{A_{1}, \ldots, A_{n}\right\}$. We first check that $\Pi$ is a robust scene:

$\Pi$ partitions $X \times Y$ since each $x \in X \times Y$ is in $\mathrm{Cl}(\cup P)=\mathrm{Cl}\left(Q_{1}\right) \cup \cdots \cup$ $\mathrm{Cl}\left(Q_{n}\right), x \in A_{j}$ for the first $j$ such that $x \in \mathrm{Cl}\left(Q_{j}\right)$; further, since each $Q_{j}$ is connected and $Q_{j} \subseteq A_{j} \subseteq \mathrm{Cl}\left(Q_{j}\right)$, each $A_{j}$ is connected, so $\Pi$ is a scene. Further, since $Q_{j} \subseteq A_{j}$ is open, $Q_{j} \subseteq \operatorname{Int}\left(A_{j}\right)$ so $A_{j} \subseteq \mathrm{Cl}\left(\operatorname{Int}\left(A_{j}\right)\right)$. If we show that $Q_{j}=\operatorname{Int}\left(A_{j}\right)$ for each $j$, then for each $j, \operatorname{Int}\left(A_{j}\right)$ must be connected and $\operatorname{Int}\left(\mathrm{Cl}\left(A_{j}\right)\right) \subseteq A_{j}$, so $\Pi$ would be a robust scene. For this it would do to show that if $N(x) \subseteq Q_{j} \cup C\left(\supseteq A_{j}\right)$, then $x \in Q_{j}$. This is certainly true if $\{x\}$ is open, since $C$ is nowhere dense. Otherwise the same argument shows that all the $(>1)$ open points of $N(x)$ are in $Q_{j}$, and if $x$ were not then for some closed separator, $x \in J \subseteq C$. But then $I(J)$, $O(J)$ both meet the open points of $N(x)$, contradicting the fact that $Q_{j}$ is connected.

Next we show that $C=\cup\{\partial D: D \in \Pi\}$. Clearly for each $j$, since $A_{j}$ is regular with interior $Q_{j}, \partial A_{j}=\mathrm{Cl}\left(Q_{j}\right)-Q_{j} \subseteq\left(X \times Y-\cup_{k \neq j} Q_{k}\right)-Q_{j}=C$; for the reverse set inclusion, if $x \in C$ then for some separator $J, x \in J \subseteq C$, thus the set $T$ of open points in $N(x)$ meets $I(J)$ and $O(J) . T \subseteq \cup P$ and since each element of $P$ is connected, $T$ must meet more than one element of $P$, say $Q_{j}, Q_{k}$. But then $N(x)$ meets $Q_{j}, Q_{j}^{c}$, so $x \in \partial Q_{j}=\partial A_{j}$.

For the converse, first note that $C$ is closed and nowhere dense. It remains to be shown that if $x \in C$ then for some closed separator $J, x \in J \subseteq C$, a fact which is clear if $n(\Pi)=1$ and which holds by 12 if $n(\Pi)=2$. Thus if this fails, it fails for a $\Pi$ with fewest elements, and for this $\Pi, n(\Pi)>2$. Note that it will do to show the result for $m$ mixed, since if $c$ is closed, find (by 14 (c)) $m \in A(c) \cap \partial A$; if $m \in J \subseteq C, J$ a closed separator then (by 14 (d)) $c \in J \subseteq C$. Thus let $m \in \partial A, A \in \Pi$; then one of the open points of $N(m)$ is in $A$, the only other is in $A^{c}=\cup(\Pi-\{A\})$, so let $m \in \partial B$. Notice that if $n \in \partial E$ is mixed, $E \in \Pi-\{A, B\}(\neq \varnothing$ since $n(\Pi)>2)$, then $n \in \partial D$ 
as well, $D \in \Pi$, and $D \neq A$ or $D \neq B$. Let $\Pi^{*}=(\Pi-\{E, D\}) \cup\{E \cup D\}$. Then $\Pi^{*}$ is robust scene with one fewer element than $\Pi$ (by 14 (a), (b)). Also $m \in U\left\{\partial F: F \in \Pi^{*}\right\}$, since the two elements of $N(m)-\{m\}$ are in distinct elements of $\Pi^{*}$. Thus by the minimality of $\Pi, m \in J \subseteq \cup\{\partial A: A \in$ $\left.\Pi^{*}\right\} \subseteq \cup\{\partial A: A \in \Pi\}=C$.

15 Proposition: Suppose $I(J), O(J)$ are regular with connected interiors, and let $J^{*}$ denote $\partial(I(J))$. Then:

(a) $J^{*}$ is a closed separator.

(b) $I\left(J^{*}\right)=\operatorname{Int}(I(J)) \subseteq I(J)$.

(c) $\left(J^{*}\right)^{*}=J^{*}$.

(d) $J^{*} \subseteq \mathrm{Cl}(J)$, and $J^{*}$ meets $\mathrm{Cl}(x)$ for each $x \in J$.

Proof: (a) We show $\{I(J), O(J) \cup J\}$ a robust scene; then $\operatorname{Int}\left(I(J)^{c}\right)=$ $\operatorname{Int}(O(J) \cup J)$ is connected, thus (a) holds by applying 12 to $A=I(J) . I(J)$ is regular, thus so is its complement, $O(J) \cup J$; they clearly partition $X \times Y$, and $\operatorname{Int}(I(J))$ is connected, thus we need only show that $\operatorname{Int}(O(J) \cup J)$ is connected.

If $x \in \operatorname{Int}(O(J) \cup J)$ it will do to show that $x$ is in the same component as $\operatorname{Int}(O(J)$ ). Let $y \in A(x) \cap O(J)$ (nonempty by [KKM]); then $x \in A(y) \subseteq$ $O(J) \cup J$, and since $y \in O(J) \subseteq \operatorname{Cl}(\operatorname{Int}(O(J))$, $\operatorname{Int}(O(J)) \cup\{y\}$ is connected. Thus $x \in N(y)$ or $y \in N(x)$; in the first case, let $z=y$, in the second, $z=x$. In both cases, notice that $N(z)$ is a connected open subset of $O(J) \cup J$, thus of $\operatorname{Int}(O(J) \cup J)$, and $x, y \in N(z)$. Thus $x \in N(z) \cup \operatorname{Int}(O(J))=$ $N(z) \cup(\{y\} \cup \operatorname{Int}(O(J)))$, again an open, connected subset of $O(J) \cup J$.

(b) By regularity, $J^{*}=\partial \operatorname{Int}(I(J))=\partial \operatorname{Int}(O(J) \cup J)$, and by 10 since $J^{*}$ is closed, $J^{*}=\partial I\left(J^{*}\right)=\partial O\left(J^{*}\right)$. All four of the above sets are open and the first two are complements in $X \times Y-J$ as are the last two, thus both $\{\operatorname{Int}(I(J)), \operatorname{Int}(O(J) \cup J)\}$ and $\left\{I\left(J^{*}\right), O\left(J^{*}\right)\right\}$, are the set of components of $X \times Y-J^{*}$, thus these sets are equal; in particular, $\operatorname{Int}(I(J))=I\left(J^{*}\right)$ or 
$=O\left(J^{*}\right)$. Suppose $x$ is the first element of $B D(X \times Y)$ not in $J^{*}$. If $x \notin J$ then by definition of $I, O, x \in O(J)$; thus in general, $x \in J \cup O(J)$ so $X \notin I(J)$ thus $x \notin \operatorname{Int}(I(J))$. But then, again by definition of $I, O, \operatorname{Int}(I(J))=I\left(J^{*}\right)$.

(c) By 10 and (a), $J^{*}=\partial\left(I\left(J^{*}\right)\right)=J^{* *}$.

(d) We first show that $J^{*} \subseteq \mathrm{Cl}(J)$ by proving that if $x \in J^{*}=\partial(I(J))$ then $N(x)$ meets $J$ : for such $x, N(x) \cap I(J) \neq \varnothing, N(x) \nsubseteq I(J)$, thus $N(x) \cap(O(J) \cup J) \neq \varnothing$. If $N(x) \cap O(J) \neq \varnothing$ then since $N(x)$ is connected and meets both components of $X \times Y-J, N(x) \cap J \neq \varnothing$, otherwise $N(x) \cap J \neq \varnothing$ anyway.

To see that $J^{*}$ meets $\mathrm{Cl}(x)$ for each $x \in J$, note that for such $x, A(x) \cap$ $I(J) \neq \varnothing$. Let $y \in A(x) \cap I(J)$; thus the connected $\{x, y\}$ meets both $I(J)$ and $I(J)^{c}$, so it meets $\partial I(J)=J^{*}$. If $x \in J^{*}$ we're done; if $y \in J^{*} \cap \mathrm{Cl}(x)$ we're done, and if $y \in J^{*}, y \notin \mathrm{Cl}(x)$ then, since $\{x, y\}$ is connected, $x \in$ $\mathrm{Cl}(y) \subseteq J^{*}$ (since $J^{*}$ is closed), and in this last case we're done.

\section{Some Subspaces for Computer Graphics.}

In this Section we consider the question of how our digital plane can be used to display graphics on a computer screen. For several reasons it is better to display only the subspace consisting of the topologically open points (which we call the "open screen" in Definition 17 below):

(i) In it, as in reality, one does not see bounding curves between adjacent regions.

(ii) It is topologically homogeneous, as the setting for a picture be.

The trace of a robust scene on the pure screen is called its display (Definition 17); Propositions 18 and 19 show that the important properties of a robust scene can be recovered from its display. Also in this Section we show how the Rosenfeld theory mentioned in the introduction can be considered 
within our theory, so that we can prove his Jordan curve theorem (Theorem 20).

16 Definition: By a Rosenfeld plane we mean a Cartesian product $\mathbf{R}=$ $\{0, \ldots, m\} \times\{0, \ldots, n\}$ (with no topology). For $(x, y) \in \mathbf{R}$, the 4 -neighbors of $(x, y)$ are the four points $(x \pm 1, y),(x, y \pm 1)$ of $\mathbf{R}$; the 8 -neighbors are the 4-neighbours and the four additional points of the form $(x \pm 1, y \pm 1)$ (the four additional points are the proper 8 -neighbors of $(x, y))$. If $k$ is 4 or 8 , a $k$-path from $(a, b)$ to $(c, d)$ is a sequence $\left(x_{0}, y_{0}\right), \ldots,\left(x_{r}, y_{r}\right)$ in $\mathbf{R}$ such that $\left(x_{0}, y_{0}\right)=(a, b),\left(x_{r}, y_{r}\right)=(c, d)$ and for each $i=0, \ldots, r-1,\left(x_{i+1}, y_{i+1}\right)$ is a $k$-neighbor of $\left(x_{i}, y_{i}\right) ;(a, b)$ and $(c, d)$ are its endpoints. A subset $S \subseteq \mathbf{R}$ is $k$-connected if for each $(a, b),(c, d) \in S$ there is a $k$-path from $(a, b)$ to $(c, d)$ contained in $S$.

A $k$-arc from $(a, b)$ to $(c, d)$ is a minimal $k$-path from $(a, b)$ to $(c, d)$. A Jordan $k$-curve is a $k$-connected set which contains exactly two $k$-neighbors of each of its elements.

Notice that for $k=4$ or 8 , a Jordan $k$-curve is precisely a $k$-path whose endpoints are identical and which contains exactly $2 k$-neghbors of each of its points. Also, a $k$-path is a $k$-arc iff it contains exactly $2 k$-neighbors of each of its nonendpoints and exactly $1 k$-neighbor of each of its endpoints.

We shall relate two theories by imbedding Rosenfeld planes in certain subspaces of our digital planes:

17 Definition: The open screen is the subspace $P=\{(x, y): x, y$ open $\}$ $\subseteq X \times Y$; the pure screen is the subspace $U=\{(x, y): x, y$ both closed or both open $\} \subseteq X \times Y$ (each with the subspace topology).

Given a robust scene $\Pi$, its associated cartoon is $C_{\Pi}=X \times Y-\cup\{\operatorname{Int}(A)$ : $A \in \Pi\}$, and its display is $D_{\Pi}=\{A \cap P: A \in \Pi\}$.

Clearly, a Rosenfeld plane can be regarded as the open screen of a digital plane; in fact, $(x, y) \rightarrow(2 x, 2 y)$ maps the Rosenfeld plane $\{0, \ldots, m\} \times$ $\{0, \ldots, n\}$ onto the open screen of $\{0, \ldots, 2 m\} \times\{0, \ldots, 2 n\}$, each given the 
COTS topology with even points open. It is natural in this open screen to consider that the 4- neighbors of $(x, y)$ are $(x \pm 2, y)$ and $(x, y \pm 2)$, and make other similar slight notational changes. (Addresses must be kept for all points even though only (open,open) points will appear on screen.) Notice that, unlike the full plane, $P$ is homogeneous.

We next show (Proposition 18) that displays and cartoons of robust scenes uniquely determine each other.

18 Proposition: Robust scenes have the same associated cartoons iff they have the same displays.

Proof: Open points are in the interiors of any sets that contain them. Thus if $C_{\Pi}=C_{\Pi^{\prime}}$, then since the interiors of elements of robust scenes are the components of the complements of their cartoons, $\{\operatorname{Int}(A): A \in \Pi\}=$ $\left\{\operatorname{Int}(A): A \in \Pi^{\prime}\right\}$, so $D_{\Pi}=D_{\Pi^{\prime}}$.

Now suppose $\Pi$ and $\Pi^{\prime}$ have the same displays. Thus if $A \in \Pi$ there is an $A^{\prime} \in \Pi^{\prime}$ such that $A \cap P=A^{\prime} \cap P$. Since $A, A^{\prime}$ are $\operatorname{regular}, \operatorname{Int}(A)=$ $\operatorname{Int}(\mathrm{Cl}(A))=\operatorname{Int}(\mathrm{Cl}(A \cap P))=\operatorname{Int}\left(\mathrm{Cl}\left(A^{\prime} \cap P\right)\right)=\operatorname{Int}\left(A^{\prime}\right)$ (with the second equality arising from the fact that each $p$ is in the closure of an open point of $N(p))$. Thus $\{\operatorname{Int}(A): A \in \Pi\}=\left\{\operatorname{Int}\left(A^{\prime}\right): A^{\prime} \in \Pi^{\prime}\right\}$, so $C_{\Pi}=C_{\Pi^{\prime}}$.

The following result shows that essentially any collection of apparently connected regions can be separated by (invisible) boundaries.

19 Theorem: Each partition of $P$ into 4-connected sets is the display of a robust scene.

Proof: Let $\Gamma=\left\{C_{0}, \ldots, C_{p}\right\}$ be a partition of $P$ into 4-connected sets. For $i=0, \ldots, p$, let $A_{i}=\mathrm{Cl}\left(C_{i}\right)-\cup_{j<i} \mathrm{Cl}\left(C_{j}\right)$ (here closures are taken with respect to the entire digital plane), and let $\Pi=\left\{A_{i}: 0 \leq i \leq p\right\}$. Since in $X \times Y$ each point is in the closure of some open points, each point must be in $\mathrm{Cl}\left(C_{i}\right)$ for some first $i$, and thus in $A_{i}$; therefore $\Pi$ is a partition of $X \times Y$. Further, for each $i, C_{i} \subseteq \operatorname{Int}\left(A_{i}\right)$, thus $A_{i} \subseteq \mathrm{Cl}\left(C_{i}\right) \subseteq \mathrm{Cl}\left(\operatorname{Int}\left(A_{i}\right)\right)$. 
Thus to show $\Pi$ a robust scene, it remains only to show that each $\operatorname{Int}\left(A_{i}\right)$ is connected:

Toward this end, let $(a, b),(c, d) \in C_{i}$, choose a 4-path $\left(x_{0}, y_{0}\right), \ldots$, $\left(x_{r}, y_{r}\right)$, from $(a, b)$ to $(c, d)$ and for $k<r$ let $\left(x_{k}^{\prime}, y_{k}^{\prime}\right)=\left(\left(x_{k}+x_{k+1}\right) / 2\right.$, $\left.\left(y_{k}+y_{k+1}\right) / 2\right)$, the unique mixed point $m$ with the property that $N(m)=$ $\left\{\left(x_{k}, y_{k}\right), m,\left(x_{k+1}, y_{k+1}\right)\right\}$. Thus, $N(m)$ meets only the region $C_{i}$ of $\Gamma$, so $m \in \operatorname{Int}\left(A_{i}\right)$, and $\left(x_{k}, y_{k}\right),\left(x_{k+1}, y_{k+1}\right) \in A(m)$, so $\left(x_{0}, y_{0}\right),\left(x_{0}^{\prime}, y_{0}^{\prime}\right), \ldots$, $\left(x_{r-1}^{\prime}, y_{r-1}^{\prime}\right),\left(x_{r}, y_{r}\right)$ is a path from $(a, b)$ to $(c, d)$ in $\operatorname{Int}\left(A_{i}\right)$. This shows that $C_{i}$ is contained in a single component of $\operatorname{Int}\left(A_{i}\right)$, which we call $Q$. Finally, if $s$ is any point of $\operatorname{Int}\left(A_{i}\right)$, then $N(s) \subseteq A_{i}$, so $N(s) \cap P \subseteq C_{i}$; thus $N(s) \cap Q \neq \varnothing$ and this shows that $N(s) \cup Q$ is a connected open subset of $A_{i}$, thus of $\operatorname{Int}\left(A_{i}\right)$, so our arbitrary $s$ is in the same component of $\operatorname{Int}\left(A_{i}\right)$ as is $C_{i}$.

Given a Rosenfeld plane, we can also imbed it in the pure screen of the digital plane $X \times X$ where $X$ is the COTS $\{0, \ldots, 2 m+2 n\}$, with even points closed, via the slant map $S(x, y)=(x+y, y-x+c)$, where $c=m+n$ if this is even, $m+n+1$ if $m+n$ is odd. In this digital plane, $(r, s)$ is pure iff $r+s$ is even, and since $(x+y)+(y-x+c)=2 y+c$, an even number, $S(x, y)$ is always pure; also $x+y, y-x+c \in\{0, \ldots, 2 m+2 n\}$. Geometrically, $S$ inserts the Rosenfeld plane at a $45^{\circ}$ angle in our pure screen, with $(0,0)$ about halfway up the left side. Also, $\left(x^{\prime}, y^{\prime}\right)$ is a 4-neighbor of $(x, y)$ iff $S\left(x^{\prime}, y^{\prime}\right) \in A(S(x, y))$, an 8-neighbor of $(x, y)$ iff there is a mixed point $(u, v)$ such that $S(x, y), S\left(x^{\prime}, y^{\prime}\right) \in A(u, v)$. For proper 8-neighbors, $(u, v)$ is the unique point between $S(x, y)$ and $S\left(x^{\prime}, y^{\prime}\right)$ on the horizontal or vertical line joining these two.

Let $J=\left\{\left(x_{0}, y_{0}\right), \ldots,\left(x_{r}, y_{r}\right)\right\}, k=4$ or 8 . If $J$ is a $k$-path, let $J^{*}$ be the image $S[J]$ of $J$ under $S$ together with the mixed points between $S\left(x_{i}, y_{i}\right), S\left(x_{i+1}, y_{i+1}\right)$ for each $i$ such that $\left(x_{i}, y_{i}\right),\left(x_{i+1}, y_{i+1}\right)$ are not 4neighbors. Notice that if $J$ is a $k$-path then $J^{*}$ is a path (in our theory); 
further, if $J$ is a $k$-arc then $J^{*}$ is an arc if $J$ is a Jordan $k$-curve (with more than three points if $k=8$ ), then $J^{*}$ is one of our Jordan curves.

It also follows from the above definition of $J^{*}$, that $J^{*}-S[J]$ contains only mixed points, and is thus empty if $k=4$. Therefore if $H$ is a 4-path and $J$ is an 8-path, and $H \cap J=\varnothing$, then $H^{*} \cap J^{*}=\varnothing$.

If $B$ denotes the border of $\mathrm{R}$, then $B$ is a Jordan 4-curve, and $I\left(B^{*}\right) \cup B^{*}=$ $S[\mathbf{R}] \cup\{(x, y):(x, y)$ mixed and $A(x, y) \subseteq S[\mathbf{R}]\}$. Finally, if $C \subseteq I\left(B^{*}\right) \cup B^{*}$ is a path, then $S^{-1}[C]$ is a $k$-path $(k=4$ if $C$ contains only pure points, 8 otherwise).

These considerations enable us to produce a topological proof of the Rosenfeld version of the Jordan curve theorem (see [Rf]) mentioned in our introduction:

20 Theorem: Let $\left\{k, k^{\prime}\right\}=\{4,8\}$. If $J$ is Jordan $k$-curve with at least 5 points which does not meet the border, then its complement in the Rosenfeld plane falls into two $k^{\prime}$-connected components.

Proof: Imbed the given Rosenfeld plane in the pure screen $U$ of a digital plane $X \times Y$ via $S$ as above. Since $J^{*}$ is Jordan curve, $X \times Y-J^{*}$ has two components, $I\left(\mathrm{~J}^{*}\right)$ and $O\left(\mathrm{~J}^{*}\right)$. We show that $S^{-1}\left[I\left(\mathrm{~J}^{*}\right)\right], S^{-1}\left[O\left(\mathrm{~J}^{*}\right)\right]$ are the two $k^{\prime}$-connected components of $\mathbf{R}-J$.

They are $k^{\prime}$-connected. If, for example, $(a, b),(c, d) \in S^{-1}\left[O\left(J^{*}\right)\right]$ then $S(a, b), S(c, d)$ are connected by an $\operatorname{arc} C$ in $O\left(J^{*}\right)$. If $C \nsubseteq I\left(B^{*}\right) \cup B^{*}$, then let $f, e$ denote the first and last points of $C \cap O\left(B^{*}\right), f^{-}$and $e^{+}$the predecessor and successor of $f$ and $e$ in $C$. Let $D=\left[(a, b), f^{-}\right]_{C} \cup\left[f^{-}, e^{+}\right]_{B^{*}} \cup$ $\left[e^{+},(c, d)\right]_{C}$, a path in $I\left(B^{*}\right) \cup B^{*}$ joining $(a, b)$ to $(c, d)$. $D$ can thus be shortened to an $\operatorname{arc} E$ from $(a, b)$ to $(c, d)$; replace $C$ by $E$. Until the end of this paragraph, the proofs of the cases $k=4$ and $k=8$ must be distinguished: If $k=4$, let $E=H^{*}$ for some 8-path, and we're done. If $k=8$ note that for any mixed point $m$ on $E$, at least one of the two pure points of $A(m)-E$ cannot be on $J^{*}$, since otherwise $m$ is on $J^{*}$ as well, contradicting the fact 
that $E \subseteq O\left(J^{*}\right)$. Replace each such $m$ by such a pure point, obtaining another path, $F \subseteq S[\mathrm{R}]$ joining $(a, b)$ to $(c, d)$ and not meeting $J^{*}$. But then $F=H^{*}=S[H]$ for some 4-connected $H$, and $H$ does not meet $J \subseteq S^{-1}\left[J^{*}\right]$. (The proof for $(a, b),(c, d) \in S^{-1}\left[I\left(J^{*}\right)\right]$, is similar. )

There is no $k^{\prime}$-arc from one of these sets to the other, for if $H$ were such, then $H^{*}$ must meet $J^{*}$, but since $J$ is a Jordan $k$-curve, disjoint from $H$, this cannot occur.

$S^{-1}\left[O\left(J^{*}\right)\right]$ is nonempty because it contains $S^{-1}(p)$ for any $p \in B^{*}$, so it remains only to show that $S^{-1}\left[I\left(J^{*}\right)\right] \neq \varnothing$. Notice that our restrictions assure us that $J^{*}$ contains more than four points, thus by Lemma $3, J^{*} \neq$ $A(m)$ for some mixed point $m . I\left(J^{*}\right)$ contains a pure point: this is immediate from the previous sentence if $I\left(J^{*}\right)$ contains only one element, but if $m, n \in$ $I\left(J^{*}\right)$ are distinct, there is an arc from $m$ to $n$ in $I\left(J^{*}\right)$, and any nontrivial arc contains pure points. 


\section{REFERENCES.}

[J] Peter T. Johnstone, Stone Spaces, Cambridge University Press, Cambridge, 1982.

[Kh1] E.D. Khalimsky (E. Halimskii), "On topologies of generalized segments". Soviet Math. Doklady 10 (1969), 1508-1511.

[Kh2] E.D. Khalimsky, "Applications of connected ordered spaces in topology". Conference of math. departments of Povolsia, 1970.

[Kh3] E.D. Khalimsky, "Ordered topological spaces". Naukova Dumka Press, Kiev, 1977.

[KKM] E. Khalimsky, R. Kopperman and P.R. Meyer, "Computer Graphics and connected topologies on finite ordered sets". To appear, Topology and its Applications.

[KMW] R. Kopperman, P.R. Meyer and Richard Wilson, "A Jordan surface theorem for three-dimensional digital spaces". To appear, Discrete and Computational Geometry.

[KR1] T.Y. Kong and A.W. Roscoe, "Continuous analogs of axiomatized digital surfaces". Computer Vision, Graphics and Image Processing 29 (1985), 60-85.

[KR2] T.Y. Kong and A.W. Roscoe, "A theory of binary digital pictures". Computer Vision, Graphics and Image Processing 32 (1985), 221-243.

[KRf] T.Y. Kong and A. Rosenfeld, "Digital topology: Introduction and survey". To appear, Computer Vision, Graphics and Image Processing.

[Re] G.M. Reed, "On the characterization of simple closed surfaces in three-dimensional digital images". Computer Vision, Graphics and Image Processing 25 (1984), 226-235. 
[RR] G.M. Reed and A. Rosenfeld, "Recognition of surfaces in three-dimen sional digital images". Information and Control 53 (1982), 108-120.

[Rf] A. Rosenfeld, "Digital topology". Amer. Math. Monthly 86 (1979), 621-630. 


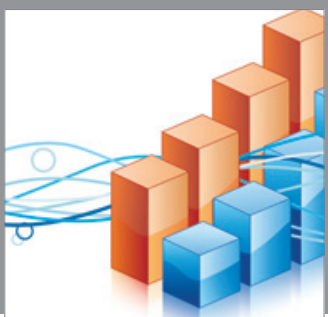

Advances in

Operations Research

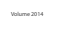

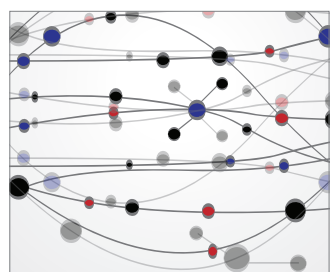

\section{The Scientific} World Journal
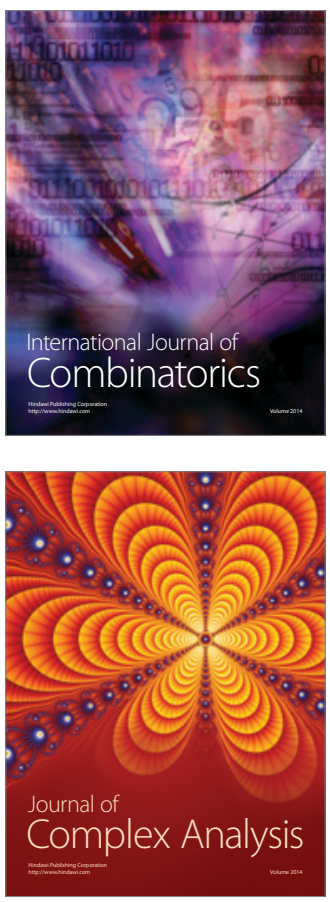

International Journal of

Mathematics and

Mathematical

Sciences
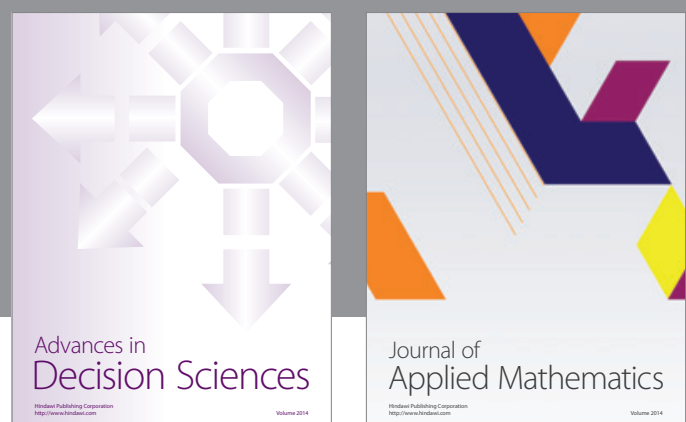

Journal of

Applied Mathematics
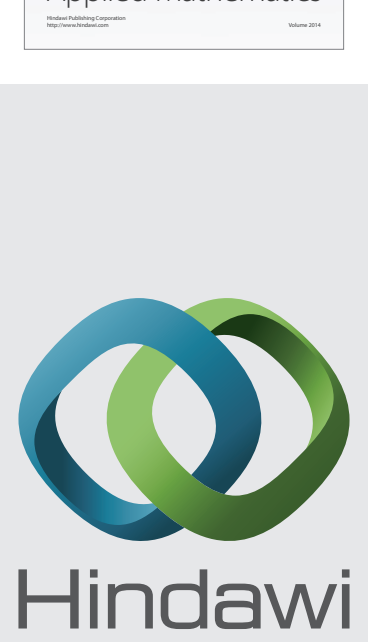

Submit your manuscripts at http://www.hindawi.com
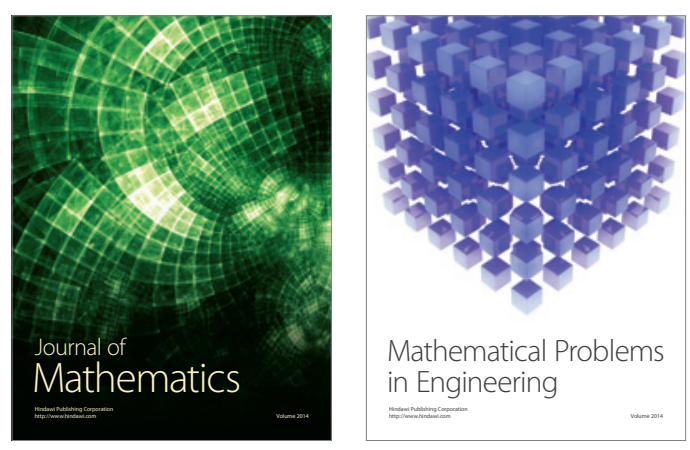

Mathematical Problems in Engineering
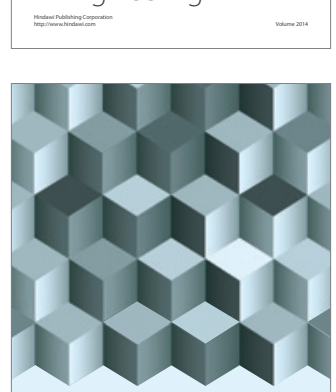

Journal of

Function Spaces
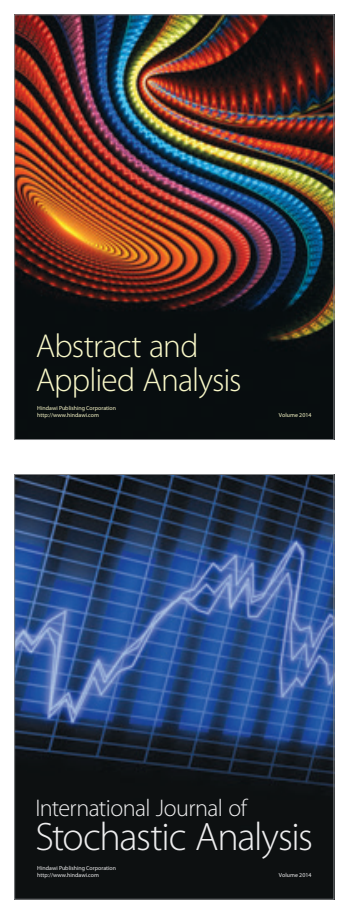

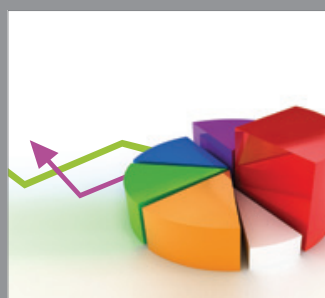

ournal of

Probability and Statistics

Promensencen
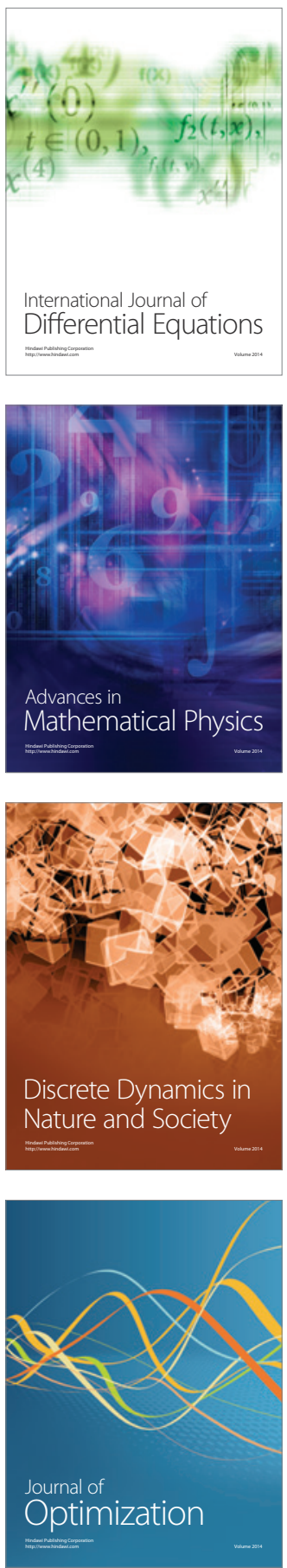